\title{
Italique
}

Poésie italienne de la Renaissance

XIV | 2011

Varia

\section{Due sonetti di Camões e la tradizione italiana}

\section{Roberto Gigliucci}

\section{OpenEdition}

\section{Journals}

Edizione digitale

URL: http://journals.openedition.org/italique/322

DOI: $10.4000 /$ italique.322

ISSN: 1663-4438

\section{Editore}

Librairie Droz

\section{Edizione cartacea}

Data di pubblicazione: 1 gennaio 2011

Paginazione: 19-46

ISBN: 978-2-600-01536-3

ISSN: 1423-3983

Notizia bibliografica digitale

Roberto Gigliucci, «Due sonetti di Camões e la tradizione italiana », Italique [Online], XIV | 2011, online dal 24 juin 2014, consultato il 01 mai 2019. URL : http://journals.openedition.org/italique/322 ; DOI : $10.4000 /$ italique.322 
Roв ER To GigLi C C I

DUE SONETTI D I A M Õ E S

E L A TRADIZIONE ITALIANA 



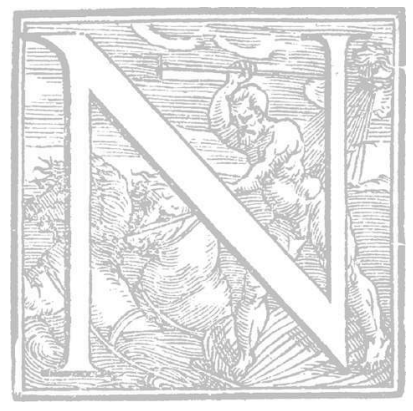

on esistono aree marginali nella geografia del petrarchismo europeo cinquecentesco, tutto è derivativo e insieme assolutamente centrale, situato sempre nel cuore dellidentità lirica moderna: l'imitatio Petrarcae. Partire dalla periferia è come farlo dal centro; anzi a volte l'identità lirica vi risuona con ancora più armonici Ma che questa identità, come ogni identità, sia una costellazione plurale e non un monolito, sia uno spazio attraversato e non un pieno omogeneo, ci pare ormai un dato ampiamente dimostrato e ribadito. ${ }^{\mathrm{I}}$

I due contributi che seguono ricercano precedenti (quasi mai candidabili a fonti) tematici e motivici di due sonetti di Luis de Camões: potrà sembrare inquisizione meramente neo-positivista, se non ci confortasse la prospettiva di verifica della tenuta europea del petrarchismo, come intertestualità e soprattutto interdiscorsività.

Uscire dall'Italia percomprendere ilpetrarchismo anche italiano èpoi una prassi ineludibile per qualsivoglia "italianista". Lo studio delle letterature nazionali in dimensione europea è quanto hanno già in molti auspicato. ${ }^{2}$ Inoltre l'ambito topico dei due sonetti (la bellezza assoluta di madonna e la sua deperibilità) ondeggia in acque significativamente extrapetrarchesche, pur all'interno della forma petrarchista; soprattutto nel secondo ambito, quello che prefigura l'incanutimento e la deprivazione, si evidenziano pulsioni aggressive e vendicative assolutamente reiette dal sistema etico-amoroso dei Rvf. Ė un ulteriore marchio di pluralità che si merita il petrarchismo soprattutto quando visto dalla specola europea, nel perfetto tenersi di coerenza e spregiudicatezza, crediamo.

\section{Beleza Ideia}

Ser ideia de beleza é ser a mesma beleza e não apenas uma mulher em quem ela se reflecte.

HERnãni CidADE

Si potrebbe dire che la straordinaria bellezza di un celebre sonetto camoniano sia già tutta nel primo verso: 
Dizei, Senhora, da Beleza ideia:

para fazerdes esse áureo crino, onde fostes buscar esse ouro fino? de que escondida mina ou de que veia?

Dos vossos olhos essa luz Febeia, esse respeito, de um império dino? Se o alcançastes com saber divino, se com encantamentos de Medeia?

De que escondidas conchas escolhestes as perlas preciosas orientais que, falando, mostrais no doce riso?

Pois vos formastes tal, como quisestes, vigiai-vos de vós, não vos vejais, fugi das fontes: lembre-vos Narciso.

Due parole sul testo, che noi diamo qui secondo l'edir: Costa Pimpão. ${ }^{3}$ Il sonetto è tramandato dal Cancioneiro de Luís Franco Correa (=LF, c. 49r) e dall'edizione delle Rimas del I668 curata da Álvares da Cunha. L'attribuzione pare certa. In LF abbiamo spesso varianti che ci sembrano qualitativamente inferiori, ripetizioni e ineleganze, che farebbero pensare a una redazione non ancora corretta o trivializzante. Ess.: 1.3 onde fostes buscar: «onde colhestes» LF, con greve ripetizione (se pure in figura etimologica) al v. 9 escolhestes; v. II que, falando, mostrais: «q Antre Robis mostrais» LF (cfr. son. 45, 3: "entre rubis e perlas doce riso»), ${ }^{4}$ ove $i$ robis, che producono coppia topica con le perle nella langue lirica, risultano più banali dellinciso falando; al v. I3 molto più povera la lezione di LF «a senrā não não no vos vejais», con un che di querulo e faticosa dieresi sul secondo não. Varianti e concieri d'autore? Forse è chiedere troppo. Nulla esclude peraltro che LF trasmettesse invece un testo più vicino all'originale e l'editore del I668 (o chi prima di lui) abbia "corretto" poi di sua iniziativa, magari anche migliorando. Recentiores non deteriores, ma può essere vero anche il contrario. O almeno una seriorità con perfezionamenti qualitativi non è necessariamente garanzia di autenticità.

L'attacco, si diceva, è memorabile, e altresi carico di tradizione, ovvero denso di arte allusiva. Si parte ovviamente dal paradigma petrarchesco: "In qual parte del ciel, in quale ydea / era l'exempio, onde Natura tolse / quel bel viso leggiadro?» (Rvf I59, I-3; si veda l'imitatio di Boscán: ¿En cuál parte del cielo, en cuál planeta). La struttura 
inquisitiva è analoga, e anche nell'architesto si evocano le «chiome d'oro» (v. 6) e gli «occhi» (v. Io); il falar e il doce riso vengono poi dalla chiusa del modello ("et come dolce parla, et dolce ride»), a sua volta, come è noto, debitrice di Orazio (Carm. I, 22, 23-24). L'andamento si ripropone ad es. al son. 220 dei Fragmenta: "Onde tolse Amor l'oro, et di qual vena, / per far due treccie bionde?), (I-2), con quel tolse che si ripercuote in buscar $e$ la vena che ritroviamo nella coppia mina-veia (e si ricordi anche Garcilaso, son. 23: «cabello, que'n la vena / del oro s'escogió», vv. 5-6); più avanti ecco le «perle» e le «dolci parole» (5-6) e infine $i$ «belli occhi» (I3).

Ma torniamo all'ingresso del pezzo camoniano. Petrarca, in apertura di In qual parte del ciel, si chiedeva (più o meno platonicamente) ove potesse essere sita l'idea che ha fornito l'exemplar da cui la natura ba riprodotto l'eccellenza creaturale di Laura. Camões va oltre, e vede nella donna il concetto stesso della bellezza, la forma medesima di ciò che è bello, offrendo una lode smisurata e iperbolica, quanto per certi versi quasi briosa (non si dimentichi che Wilhelm Storck rinveniva un'ironia galante nel sonetto, a suo parere rivolto a qualche dama frivola ed egocentrica). ${ }^{6}$

Tuttavia il verso di apertura camoniano è da inserire nella lunga trafila che parte e si diparte dall'attacco appunto petrarchesco del son. I59. Protagonisti di tale vicenda sono soprattutto nel Cinquecento due celebrati sonetti di Bembo e di Della Casa: Se stata foste voi nel colle ideo del primo ${ }^{7}$ e La bella greca, onde 'l pastore ideo del secondo. ${ }^{8}$ In entrambi c'è il topos della bellezza di Venere prescelta da Paride sul monte Ida, e dell'ulteriore superamento in bellezza da parte della donna amata dai poeti. Motivo che compariva pure nell'Orlando furioso (XI, 70: "Se fosse stata nelle valli Idee») e in modelli classici (Properzio, Ovidio). Gutierre de Cetina vi dedica anch'egli un sonetto, rivolto A la Condesa Laura Gonzaga:" "Laura, si cuando en la gran selva Idea / hizo el juicio aquel pastor troiano» ecc., con le terzine evocanti il paradigma del pittore Zeusi: se l'artista che raffigurò la bellezza ideale di Elena cogliendo bellezze parziali e ricomponendole (come un poeta che opera la mellificatio) avesse preso a modello soltanto la dedicataria del sonetto, avrebbe ottenuto maggiore bellezza e grazia e soprattutto "más ser», 'più essenza', avrebbe mostrato piu direttamente e integralmente l'essenza stessa della bellezza, il suo compimento. «iTanto el cielo de vos se satisfiro!», conclude esclamativamente 
il Cetina. Dunque la donna è oggetto del compiacimento divino (espressione come è noto biblica). E quindi, per quanto supremamente perfetta, è comunque creatura, non è assolutamente autonoma come la Senhora camoniana.

Il locus del confronto ideo non è certo assente dalla rimeria petrarchista italianizzante del rinascimento lusitano; segnaliamo soltanto Diogo Bernardes: "Se quando vio as Deosas no monte Ida / o Troiano pastor, tambem vos vira, / Venus dalli tão leda não partira / c'o preço, por quem foi Troia perdida» (Rimas varias Flores do Lima son. XXII, I-4). ${ }^{\text {IO }}$ Ma tornando al quadro italico, il giudizio di Paride era già ben presente in lirici del Quattrocento, fra cui citiamo soprattutto Panfilo Sasso: «Se fosse stata al tempo del Troiano / questa d'ogni beltade exempio e idea» (ed. Malinverni ${ }^{\text {I }}$ son. 27). In Sasso la donna risale il grado da esemplata a esemplare, diventa essa stessa idea d'ogni bellezza, e la strada per Camões è aperta. Anche tenendo conto che fra $i$ sonetti di Panfilo ve n'è un altro sullo stesso tema, Fra mille exempi di beleza Iove (60) in cui ritroviamo gli occhi lucenti, $i$ capelli d'oro e il «dolce riso». E possiamo evocare anche il son. Nympha che spargi a l'aura el bel crin d'oro (32), dalla struttura interrogativa e con la chiusa: «onde sei certo idea, / ché mortal forma non è sì soprana». Panfilo Sasso potrebbe essere infatti, da più riscontri, una lettura di Camões: si pensi solo alla ricorrente metafora dei fili d'oro per $i$ capelli, non cosi comune ${ }^{\mathrm{I} 2}$ e cara al Camões lirico ed epico (vd. infra), anche se certo implicitamente autorizzata da Rvf 198 I-2 ("L'aura soave al sole spiega e vibra / l'auro ch'Amor di sua man fila e tesse»)) e presente in Ariosto lirico.

Naturalmente si potrebbero fare ulteriori esempi quattrocinquecenteschi, più o meno prossimi all'oltranza camoniana. Di osservanza petrarchesca, e quindi panegiristi moderati di una donna esemplata e non esemplare assoluto, sono ad es. $i$ sgg.: Fabio Galeota, canz. Ecco loco deserto vv. 40-43: "Da la più cara Idea, / di quante eran nel cielo / tolse natura il più leggiadro esempio, / et formò te mia Dea» (Libro quinto delle rime di diversi illustri signori napoletani, Venezia I555, p. I22); Chiara Matraini: «e da la Idea / più bella in Ciel, da la più eccelsa sede / tolse, formando in voi l'etterno essempio», "Da la più eccelsa Idea, dalla più chiara / luce beata che nel Ciel si mostra, / venne fra noi la bella anima vostra», "dalla più bella e chiara Idea / vi fe' Natura essempio» (ed. Rabitti ${ }^{\mathrm{I}}$ PD. 52, 207, 2I0); Berar- 
dino Rota: "Quando Dio tolse da la propria idea / quel bel ch'entro e di fuor vi orna e veste» (son. XCI ed. Milite ${ }^{\mathrm{I}}$ ). Più vicini all'iperbole encomiastica di Camões sono invece, e. g., Bernardo Tasso: «Specchio di vero ben, di vero onore, / Idea de la beltà celeste e diva» (Libro secondo degli Amori 80, I2-I3); ${ }^{\text {I5 }}$ Varchi, Immortal donna, anzi mortale dea: "La vostra unica in terra alma bellezza / di quante furo e fien beltati idea» (Sonetti spirituali parte III, son. LXVIII), ${ }^{6}{ }^{6}$ ecc. Ma certo l'autoprodursi estetico, il fazer-se indipendente della signora camoniana è segno di tale oltracotanza da virare la lode in implicito biasimo e quasi accusa di empietà. Cosa che non può darsi nella gran parte dei modelli petrarchisti, per lo più platonico-cristiani. Come, per fare un ultimo esempio, nelle ottave di Bernardino Tomitano, ospitate dal Libro secondo di Rime di diversi (I547), forse presenti al nostro: "Onde tolse Natura il bel thesoro, / di cui vi fe' si ricca et si possente? / Et zaphiri, et coralli et perle et oro, / di che andate si altera et si lucente? / [..] In qual parte del ciel la cura eterna / tolse l'idea di voi, l'essempio adorno?» (c. 43r).

Gli arcimodelli Bembo e Casa mettevano in gioco un circolo platonicomitologico che rimandava dall'idea all'aggettivo ideo, contaminando il concetto supremo di bellezza con la vicenda del pastore ideo che premiò Afrodite e quindi proiettando sulla perfezione di madonna la perfezione assoluta della dea stessa della bellezza. Questo cortocircuito poteva essere assente dalla consapevolezza di Camões? Forse no, e allora implicitamente Dizei, Senhora, da Beleza ideia non può essere scompagnato da quel mitologema che è citato apertamente ad esempio in Lusíadas II, 35, 3-4: "Se Ih'apresenta assi como ao Troiano, / na selva Ideia, já se apresentara». D'altra parte, ancora citando Camões stesso, non sarà soltanto casuale e strumentale che la suprema Idea empirea rimi proprio con Citerea nei celebri versi in castigliano a conclusione dell'egloga prima: «ahora embevecido estés mirando / alla en el Empireo aquella Idea / que el mundo enfrena y rige con su mando; / / abora te possuya Citerea / en su tercero asiento». ${ }^{17}$ E ancora, sarà da ricordare che nel celebrato son. Transforma-se o amador ${ }^{18}$ abbiamo semideia in rima con ideia: la prima risiede nel pensiero come idea, idea formante, modello formale e produzione di forma nella "materia semplice" del poeta innamorato. Si noti che la rima ricca inserisce materialmente l'ideia nella semideia, rendendo quasi obbligante la sovrapposiżone fra paradigma e (semi)divinità. Infine rammentiamo 
che la forma latineggiante Deia rima con Citereia in una delle importanti ottave del primo libro del poema, dove si chiariscono $i$ rapporti delle divinità con gli eroi portoghesi (ott. 34, I-3): il cortocircuito ideiaIdeia-deia-Citereia ${ }^{19}$ mi pare dunque indubitabile nel pensiero linguistico della poesia camoniana.

Allora nel nostro sonetto abbiamo ideia che reclama una criptica presenza dell'attributo Ideia, ovvero una ideia da Beleza che va a coincidere con la beleza Ideia, sottintendendo cosi la scena chiave nel bosco sull'Ida e quindi facendo sorgere dietro madonna la Deia-Deusa stessa della beltà, ovvero la fermosa Dione la cui descrizione nel poema è non dissimile da quella di Dizei, Senhora: «Os crespos fios d'ouro s'esparziam» ecc. (II, 36), che rimanda a sua volta al son. IO Quem pode livre ser, gentil Senhora, v. Io: "fios crespos d'ouro». ${ }^{20}$ Se restiamo a osservare $i$ capelli e gli altri elementi della raffigurazione femminile, più di un dato avvicina il sonetto a quello attribuito nel Cancioneiro de Fernandes Tomás [FT] a Fernão Rodrigues (Roiz) Soropita, Esses cabelos louros escolhidos (presente anche nell'edizione camoniana del 1668): l'oro dei capelli, gli occhi, la grazia divina che falando promana dalla dama ecc.; sempre di Soropita e sempre in FT si veda anche Cabelo em ricos laços ordinado, in una serie che deriva dal modello bembiano Crin d'oro crespo, come bene evidenzia Rita Marnoto, inserendovi prima ancora che Dizei, Senhora l'altro sonetto camoniano Ondados fios d'ouro reluzente. ${ }^{2 \mathrm{I}} \mathrm{E}$ se volessimo regestare le occorrenze effictivemetaforiche di oro-capelli, luce-occhi, perle-denti nella tradizione dovremmo riempire pagine con elenchi fantastici come quelli che profonde Faria e Sousa. ${ }^{22}$ Fermiamoci però a un confronto interno al corpo rimico camoniano per verificare lo scarto di Dizei, Senhora. Il sonetto Quando da bela vista e doce riso ${ }^{23}$ racconta dell'estasi paradisiaca del poeta davanti alla donna del cui aspetto e riso egli si nutre con lo sguardo; si tratta di un accesso al divino nell'excessus mentis contemplativo, con la consapevolezza che non è possibile fundar-se nella lode impraticabile. La terzina finale suona: "Que de tanta estranheza sois ao mundo, / que não é d'estranbar, Dama excelente, / que quem vos fez, fizesse Céu e estrelas». Prescindendo al momento da fonti e intertesti di questo sonetto (Faria e Sousa ne offre copia, come sempre), sia sufficiente osservare che lo stupor mundi rappresentato dalla Dama si razionalizza considerandolo creazione di Dio, che fece anche cielo e 
stelle. Dunque la lode della creatura si connette con la lode del Creatore. Cosi anche nell'ode Pode um desejo imenso, fra le composizioni più spiritualizzanti del nostro, "a luz alta e severa» è «raio da divina fermosura». ${ }^{24}$ Diversamente in Dizei, Senhora la donna, ribadiamo, è creatrice di se stessa, si è formata come essa ha voluto, è dea a se stessa, perfettamente autosufficiente nel rappresentare in sé come soggetto e oggetto il sopraumano. Proprio qui sta la sottile malignità della lode smodata: l'induzione di un'autonomia della SenhoraDeusa da Dio la fa ricadere nel sospetto di praticare gli «encantamentos de Medeia), e quindi la situa nella sfera diremmo "diabolica" della magia e del mito. Anche in un altro sonetto fra quelli dell'edizione I668, Quem presumir, Senhora, de louvar-vos, l'iperbole enlogiastica sale di molto, partendo nelle quartine dal topos della ineffabilità assoluta rispetto a ogni tentativo di lode, per arrivare a un eccesso quasi irriverente: «que vossa fermosura eu imagino / que Deus a Ele só quis comparar-vos». ${ }^{25}$ Però l'omaggio supremo si ferma qui a Dio, il quale scelse un'unica creatura per esprimere appieno la propria immagine e somiglianza, ma comunque restandone il creatore. Invece in Dizei, Senhora, Dio non c'è proprio, la scena della creazione è una solitudine di auto-creazione, e in questa solitudine c'è il rischio del male e della sventura.

Abbiamo detto dell'ombra luminosa e implicita di Venere dietro alla Senhora del sonetto. D'altra parte la lirica è tutta tramata di tessere mitiche esplicite, dall'evocazione di Medea a quella di Narciso. Sono peraltro mitemi piuttosto cupi, magici, ominosi e mortuari. Che culminano nel finale rischio-presagio di un'autodistruzione della dama prigioniera del proprio stesso circuito, innamorata di se stessa (cioè della propria perfetta creazione) come Narciso appunto. Un uso del mito narcissico all'interno della lirica d'amore spesso in chiave aggressiva, vendicativa, è documentato. Si pensi, oltre che ovviamente a Rvf 45 ("Certo, se vi rimembra di Narcisso, / questo et quel corso ad un termino vanno, / benché di si bel fior sia indegna l'erba», I2-I4), a una "dispersa" del Petrarca, il son. Lasso, com'io fui mal approveduto: "Signor, fa vaga lei del suo bel viso / da poi che fuor di sé non sente ardore, / rinnova in lei l'esempio di Narciso») (I2-I4, presente nell'aldina del Is I4 e nella giuntina del Is22 delle Rime di Petrarca). ${ }^{26}$ Tebaldeo: "Spèchiate nel superbo e bel Narciso / che per voler usar troppo durezza / da l'amor de sé stesso alfin fu occiso» (estravagante 
708, 6I-63 ediz. Marchand ${ }^{27}$ p. I049: ternario tràdito dal ms. 7-2-3I della Capitulary Colombina di Sevilla). Boiardo, Amorum libri I79, 49-52: "Non vi spechiati a questa fonte il viso, / ché morte occulta vi darà di piglio: / in quel fioreto candido e vermiglio / sol per mirarsi se cangiò Narciso» (edir. Zanato ${ }^{28}$ pp. 577-578: si tratta di una canzone contro l'amore, penultimo pezzo del canzoniere boiardesco, che insiste sulla raccomandazione di fuggire: "Fugeti mentre il senso non vi ̀̀ tolto», v. 45, ecc.). Parabosco, madr. 19, 9: «E siavi essempio il bel crudo Narciso» (ediz. Longo ${ }^{29}$ p. 73). E cosi via. Insomma, evocare Narciso significa preconizzare astiosamente la rovina della donna sans merci autoreferenziale. ${ }^{30}$

Quanto a Medea, spesso in coppia con Circe viene nominata dai petrarchisti come equivalente mitico della donna amata e crudele. Soltanto un esempio, fra $i$ più vagamente prossimi al concetto camoniano: "Donna è nel mondo che m'attrista e incende / con si fero liquor, si forti incanti, / che di Circe e Medea non più s'intende. / / L'erba son chiome e i duo bei lumi santi / che versan tal virtù, ch'uom che ne beva / altro divien da quel ch'egli era innanti» (Luigi Alamanni, elegia Ben mi credea poter senz'altra cura, ediz. Raffaelli, ${ }^{\text {I }}$ I $\not p$. $47^{-}$ 48). Tuttavia anche qui l'incanto di Medea si esercita sull'amante, mentre in Camões la Dama è di nuovo, come dire, Medea a se stessa, se è vero che si procura da sé l'imperiale respeito, non ricevendolo dalla divinità.

In conclusione, possiamo ribadire l'estraneità, se non opposizione, di questo sonetto camoniano alla dimensione platonica e neoplatonica. La Senhora si sottrae infatti al circuito creatura-creatore, si distanzia dall'asse verticale ${ }^{32}$ per cui dalla corporeità umana si sale alla sfera divina. La Senhora è autonoma dal processo circolare secondo cui l'amore divino crea la creatura e questa risale attraverso $i$ gradi dell'amore al creatore: la Senhora si è fatta da sé, al limite forse per incanto diabolico; ha originato la propria bellezza - che è idea di bellezza, quindi archetipo stesso, non esemplato ma paradigmatico cogliendo chissà da quali materie terrestri remote e supreme gli elementi costitutivi della sua persona perfetta. Per questa oltranza di SenhoraDomina-Deusa, per questa sua autosufficienza meravigliosa ma quasi blasfema, pende su di lei una minaccia, la minaccia dell'autodistruzione, la minaccia dello specchio di Narciso. Non è solo il rischio comune a tutte le altre sdegnose amate dai poeti e non riamanti. Ė un 
pericolo più grande, che si addice alla grandiosità di chi non è sottomessa neppure a un dio creatore.

\title{
2. Ouro $>$ prata
}

\author{
Il desiderar bruttezza \\ nella donna che s'ama di cuore, \\ non è affetto di nobile e vero amante.
}

Alessandro TAssoni

Il motivo cosiddetto dell'amata incanutita, ${ }^{33}$ alimentato da più o meno sottile risentimento e spirito di vendetta, ha una declinazione malinconica ma pure alfine affilata in un sonetto di Camões:

Se as penas com que Amor tão mal me trata

quiser que tanto tempo viva delas

que veja escuro o lume das estrelas

em cuja vista o meu se acende e mata;

e se o tempo, que tudo desbarata,

secar as frescas rosas sem colhê-las, mostrando a linda cor das tranças belas

mudada de ouro fino em bela prata;

vereis, Senhora, então também mudado

o pensamento e aspereza vossa, quando não sirva já sua mudança.

Suspirareis então pelo passado, em tempo quando executar-se possa em vosso arrepender minha vingança.

L'attacco del pezzo è simile a quello di un son. del corpus camoniano spurio, ${ }^{34}$ attribuito al Duque de Aveiro nel Cancioneiro Fernandes Tomás [FT I54v], mentre nel Cancioneiro de Luís Franco Correa [LF I39v] non ba indicazione di autore: Que fiz, Amor, que tão mal me tratas? Nonostante l'apertura analoga, il sonetto del Duque de Aveiro procede tematicamente in ambito del tutto diverso da quello di Camões (il quale, non si dimentichi, dedicò al Duque de Aveiro, João de Lencastre, l'ecloga A rústica contenda desusada). 
Se as penas si legge, oltre che nelle prime due edizioni a stampa, nel canzoniere LF in due versioni piuttosto differenti, soprattutto per quanto concerne $i$ versi I-2, come illustra dettagliatamente l'edizione critica di Azevedo Filho. ${ }^{35}$ L'autoria camoniana è ritenuta comunque incontroversa.

Le ascendenze di questo sonetto di Camões, possibili o solo probabili, sono illustri. Nelle Rime di Bembo del Iszo troviamo un sonetto come il seguente (che sarà riproposto nelle edizioni posteriori): $3^{36}$

O superba e crudele, o di bellezza ${ }^{37}$ e d'ogni don del ciel ricca e possente, quando le chiome d'or caro e lucente saranno argento, che si copre e sprezza,

e de la fronte, a darmi pene avezza, l'avorio crespo, e le faville spente, e del sol de' begli occhi vago ardente scemato in voi l'onor e la dolcezza,

e ne lo specchio mirarete un'altra, direte sospirando: «㐫, lassa, quale oggi meco pensier? perché l'adorna mia giovenezza ancor non l'ebbe tale? con questa mente o 'l sen fresco non torna? Or non son bella, alora non fui scaltra».

Il sonetto parafrasa ora più ora meno fedelmente un carme di Orazio (Odi IV, IO, che per la precisione era rivolto ad un ragaz:20) ${ }^{37}$ e risente indubbiamente di Petrarca, Rvf $12:^{38}$

Se la mia vita da l'aspro tormento si può tanto schermire, et dagli affanni, ch'i’ veggia per vertù degli ultimi anni, donna, de' be' vostr'occhi il lume spento, e i cape' d'oro fin farsi d' argento, et lassar le ghirlande e i verdi panni, e 'l viso scolorir, che ne' miei danni allamentar mi fa pauroso et lento:

pur mi darà tanta baldanza Amore ch'i' vi discovrirò de' miei martiri qua' sono stati gli anni e i giorni et l'ore; 
et se 'l tempo è contrario ai be' desiri, non fia ch' almen non giunga al mio dolore alcun soccorso di tardi sospiri.

L'aggressività di Bembo scavalca indietro il modello petrarchesco recuperando gli stati d'animo dei poeti latini come Orazio, ma anche Ovidio, che augurava una cariosa senectus alla donna ingannatrice (Amores I, I2, 20-30), e gli elegiaci in generale, per $i$ quali la recriminazione violenta nei confronti dell'amata è più che lecita, mentre non sarebbe concepibile nell'universo cortese-petrarchesco. Su questo ci siamo soffermati altrove. ${ }^{40}$

D'altra parte anche Tebaldeo - quel Tebaldeo che il Bembo andava seppellendo - in un sonetto della vulgata imitò il medesimo Petrarca situando già il topos in un'area di ostilità francamente feroce:

Se avien che 'l ciel me dia viver tanti anni che quella treza d'or veggia d'argento, e il vermiglio color del viso spento, e il corpo in altra scorza e in altri panni, ricordarote tanti oltragi e inganni; e come hora tu ridi del mio stento, cussì anch'io riderò lieto e contento del tuo color deforme e de' toi danni.

Né temerò questi toi fieri sguardi, ché gli occhi non aràn più foco hormai e Amore altrove temprarà soi dardi.

Alhor di sdegno il specchio spezarai; ma sì forte me struggi e sì forte ardi, che quel giorno veder non credo mai. ${ }^{4 \mathrm{I}}$

Nonostante la nota sfiduciata finale, il poeta riderà bene perché riderà ultimo, e prefigurazione più aspra non si poteva dare. Va qui però ricordato, anche se è cosa nota, che pure Boccaccio aveva rimato sul motivo, con tre sonetti (Rime XLIII, XLIV, I 3$)^{42}$ dei quali almeno $i$ primi due si segnalavano per una vis vendicativa molto aspra massime sul piano lessicale; riportiamo il son. XLIV:

S'egli avvien mai che tanto gli anni miei lunghi si faccin, che le chiome d'oro 


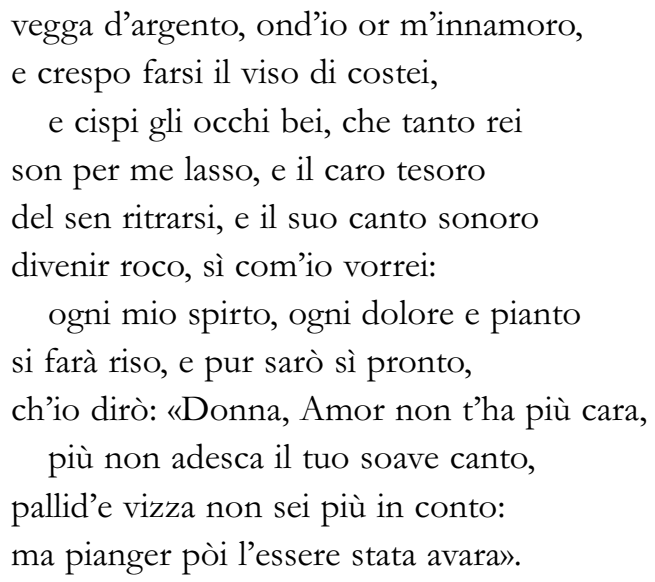

L'acredine boccacciana si misura in certe scelte lessicali quali cispi o vizza, che appartengono di diritto all'ambito cosiddetto comicorealistico, o in immagini da iconografia orrida come il ritrarsi del seno. E la ritorsione impietosa è marcata dal riso del poeta (poi tebaldeano) in cui si converte il lamento durato per una vita, nota rabbiosamente allegra che concludeva anche il son. XLIII: "Oh, s'io potesse creder di vedere / canuta e crespa e pallida colei, / che con isdegno nuovo n'è cagione! // Ch'ancor la vita mia di ritenere, / che fugge, a più poter m’ingegnerei, / per rider la cambiata condizione» (vv. 9-I4). Le forme del comico nella lirica d'amore per Boccaccio sono convenienti giusta il modello ovidiano e, diversamente, quello petroso dantesco. In Petrarca invece la senescenza è il momento di una ulteriore virtuale dolcezza e dell'estremo sfinito soccorso.

Ritornando al Cinquecento e all'area veneta, Domenico Venier, il principe degli artificiosi, si pone nella trafila sulle orme bembiane: ${ }^{43}$

O più ch'altra giamai cruda, e rubella d'Amor, a cui ben fu largo e cortese d'ogni suo dono il Ciel, ch'a farvi intese la più vaga del Mondo, e la più bella;

quando i crin biondi, e l'una, e l'altra Stella fian senza l'oro, e le faville accese, e mille rughe havran le guancie offese, ch'avorio terso fa l'età novella;

e ne lo specchio quasi in poco d'hora vedrete un'altra, invan con gli occhi molli 
direte: «Hor qual pensier meco soggiorna?

Perché tal non l'hebb'io giovane ancora?

O con questa mia mente il bel non torna?

Oggi lassa non posso, allor non vollì.

Un altro veneziano, peraltro frequentatore del salotto Venier, come Antonio Mezzabarba, il cui rilievo è ora finalmente acclarato, ${ }^{44}$ aveva pure lui replicato il gesto bembiano, aggiungendovi un ulteriore pannello; nelle sue Rime edite dal Marcolini nel I536 si leggeva infatti il seguente dittico:

O pur crudel, e per tanti e sì cari

don che pò mai natura e'l ciel qui dare

meravigliosa al mondo e senza pare, se non fosser di sé pur troppo avari,

quando i crin d'oro farsi bianchi e rari

vi mirarete, e l'avorio crespare

del bel viso, i vermigli fior mancare,

spento il dolce e l'ardor dei lumi chiari,

né più vi renderà lo specchio adorna,

direte in van dogliosa, e dei miei danni

forse, ne' quai superbia or vi trastulla:

«Qual oggi è 'l mio pensier? Perché fanciulla

non l'ebbi io tal? Ahi, perché a me non torna

con questo animo il bel de' miei primi anni?».

Di quante mai bellissime già foro più ricca e avara, e voi lumi possenti nel maggior mal a darmi util ristoro, et a' miei danni sì bramosi e intenti, non sempre durerà lo avorio e l'oro, le bianche perle e li robini ardenti: prestato vi è quel dolce e bel tesoro, che sol pò far i miei desir contenti.

Va ognor mancando in voi tanta bellezza, tosto i biondi capelli caderanno, e del vivo color l'alma vaghezza.

Vendetta fia del mio sì lungo affanno la sì sprezzata e povera vecchiezza; ma vostri rei pensier che fin avranno? 
È assai avvincente reperire in queste Rime dell'amico di Aretino e prossimo a un poetare apparentemente anti-bembiano (ma anche prebembiano, almeno per certi componimenti giovanili, e comunque riverente ufficialmente $)^{45}$ la stessa traduzione-rielaborazione del carme oraziano che Bembo aveva rilanciato: Mezzabarba è solito "imitare" $i$ latini, massime i piu lascivi come Catullo, ma con tale operazione omaggia anche lo stesso Bembo. Si noti ancora, al v. 6 del primo sonetto, come il consueto attributo crespo/a, a volte elogiativo della chevelure, a volte indicativo dell'ingrinzirsi della pelle, sia qui animato in verbo (crespare), non frequente nella lirica petrarchista; ${ }^{46}$ ne serba traccia Baldassarre Stampa quando verga un suo sonetto da inserire anch'esso senz'altro nella tradizione che qui stiamo in parte ricostruendo:

Se v'accorgeste del fuggir de l'ore, e come il tempo con l'usato artiglio crespar le guance e 'l candido e vermiglio suol tramutar in pallido colore,

e 'l vago agli occhi, al viso tôr l'onore, usareste altro modo, altro consiglio, madonna, e con sereno e lieto ciglio omai trareste me di doglia fore.

Deh, non v'insuperbite a l'esser bella. Cadeno i gigli; e voi direte alfine, dannando il giovenile orgoglio altero:

«Lassa, quanto mutata io son da quella! O saggio amante! ahi bel perduto crine! Invan fui bella, e invan muto pensiero"». ${ }^{47}$

Il modello oraziano è qui incrostato di almeno due vistose citazioni virgiliane (alba ligustra cadunt, quantum mutatus ab illo!), segnalate anche da Luigi Baldaci nei suoi memorabili Lirici del Cinquecento. ${ }^{48}$ Infatti la musa del fratello di Gasparina è nativamente melanconica soprattutto nellindugiare sul motivo della fuga temporis: ecco che all'artiglio del tempo del v. 2 possiamo accostare la sabbia della clessidra del son. Il vostro dono prezioso e caro, sempre di Baldassarre, cantore elegiaco del viver frale e della brevità dell'ora. ${ }^{49}$

Con assai minore delicatezza nell'ultima terzina del secondo sonetto il Mezzabarba poneva in rilievo trionfalmente l'esplicitazione della ven- 
detta. La parola-tema vendetta ${ }^{50}$ ̀̀ ancora ben patente anche al primo verso di un sonetto di Torquato Tasso nella raccolta per Lucrezia Bendidio:

Vedrò da gli anni in mia vendetta ancora far di queste bellezze alte rapine, vedrò starsi negletto e bianco il crine che la natura e l'arte increspa e dora;

e su le rose, ond'ella il viso infiora, spargere il verno poi nevi e pruine: così il fasto e l'orgoglio avrà pur fine di costei, ch'odia più chi più l'onora.

Sol penitenza allor di sua bellezza le rimarrà, vedendo ogni alma sciolta de gli aspri nodi suoi ch'ordia per gioco;

e, se pur tanto or mi disdegna e sprezza, poi bramerà, ne le mie rime accolta, rinnovellarsi qual fenice al foco.

Qui il topos aggressivo mantiene intatta la sua spregiudicatezza rispetto al pudore petrarchesco e in più ostende l'elazione stilistica, la predilezione per una gravità ben temperata propria della lirica amorosa secondocinquecentesca, ${ }^{5}$ dove Tasso è principe, in una dorsale che dall'ultimo Bembo attraverso il Casa perviene senz'altro a lui, com'è noto. Il son. seguente, Quando avran queste luci e queste chiome, è invece più moderato, anzi sopprime proprio il moto vendicativo e insiste sull' amore inestinguibile e soprattutto sull'inestinguibilità del canto poetico: «mostrerò ne gli alti carmi / le tue bellezze in nulla parte offese» ( $v v$. IO-II). Naturalmente sono stati in tanti ad esercitarsi sul carme oraziano a Ligurino; potremmo citare anche un lirico originale e solo un poco marginale come il Coppetta Beccuti, con la sua ottava relativa: ${ }^{2}$

Quando sarà ch'io veggia ai giorni miei cadere il fiore al tuo bel viso adorno e che, cangiata, aimè! da quel ch'or sei, sospiri e dichi al fido specchio intorno: «Perché non volsi io già quel ch'or vorrei, o con questo voler bella non torno?» Cangia dunque pensier, mentre che puoi, ed accorda cogli anni i disir tuoi. 
Spostandoci in area iberica, molto più fedele a Bembo è la «tradución»s3 di Fernando de Herrera:

O sobervia i cruel en tu belleza, cuando la no esperada edad forçosa del oro, qu'aura mueve deleitosa, mude 'n la blanca plata la fineza; i tiña 'l roxo lustre con flaqueza en l'amarilla viöla la rosa, i el dulce resplandor de luz hermosa pierda la viva llama i su pureza;

dirás (mirando en el cristal luziente otra la imagen tuya): «Este desseo ¿por que no fue 'n la flor primera mia?

¿Por que, ya que conosco el mal presente, con esta voluntad, con que me veo, no buelve la belleza que solia?».

Il testo è presente nelle Anotaciones di Herrera alle opere di Garcilaso de la Vega, ${ }^{54}$ in particolare nelle pagine di commento allo stupendo sonetto En tanto que de rosa i açucenass sul rapido sfiorire della rosa e sul carpe diem. Herrera adduce numerose "fonti", fra cui la celebre elegia di Ausonio Ver erat, nonché Plinio, Seneca, e l'Orazio sopra citato, di cui offre per esteso le "traduzioni" di Bembo, di Venier, la propria, e ne aggiunge una di Tommaso Mocenigo, che riportiamo subito:

O sempre a me più disdegnosa e fiera, ch'haveste in don dal ciel alma beltade, né vi pensate mai che presta etade a voi torrà quel pregio, ond'ite altiera; quando l'aurata chioma, ch'hor leggiera si volve a l'aura, hor si rincrespa, hor cade, fia chiuso argento, e le vaghezze rade co' bei color smarrite, e giunte a sera; direte «Ohimè», qual hor dentro a lo speglio cangiata vi vedrete in altro aspetto, «Qual pensier mi si desta hoggi nel petto? 
Perché 'n mia gioventù non scorsi il meglio? O con animo tal perché non torna quel biondo crine, e quella guancia adorna?». ${ }^{56}$

Restando nel grande «mar de la erudición», non si può mancare di dare una sbirciata fra le pagine del pletorico commento di Manuel de Faria e Sousa alle rime camoniane, e in particolare al son. Se as penas. ${ }^{57}$ Se ne ricava una sterminata quantità di suggestioni intertestuali, che va ben oltre gli autori da noi citati finora. Cosi a Bembo, Tebaldeo, Boccaccio, Petrarca, Mocenigo ecc. troviamo affiancati lacerti dalle rime di Amalteo, Marmitta, Minturno, Rota, Paterno, Guarini, Camillo Besalio, Varchi e numerosi altri, oltre a spagnoli e ovviamente latini. Si tratta di frammenti non sempre cospicui, ma a indubbia testimonianza che il motivo è res nullius, ovviamente soprattutto nella declinazione del carpe diem e del collige florem. Il grande erudito secentesco è cosciente che tanta copia di testi offre una moltiplicata ipotesi d'interdiscorsività, oltre e più che d'intertestualità: "Esso todo es quanto a lugares assi en general como en particular, que mi $P<$ oeta $>$ pudo ver para imitarlos, o en que sin ver a todos concurrio con ellos». ${ }^{58}$ Tuttavia Faria e Sousa ritiene in conclusione che $i$ due sonetti indubitabilmente (《sin duda») noti a Camões siano stati Se la mia vita di Petrarca, naturalmente, e Se avien che'l ciel di Tebaldeo, anche se l'immagine delle trecce d'oro che si fanno argento, presente in Tebaldeo e in Camões, epperciò probante per Faria, non sembra certo un unicum.

Il tema fu affrontato direttamente anche da Diogo Bernardes (Flores do Lima son. XXIII):

Se poder tanto à morte defenderse a vida, que por vos deve estimarse, que veja em vossos olhos apagarse a luz, que faz o sol escurecerse, e o ouro dos cabellos converterse em branca prata, o rosto descorarse, de tal maneira em fim tudo mudarse, que mais ousadamente deixe verse:

então firme en mudanças tão continas vereis como não amo, nem receio de vos o que não pode ter firmeza, 
mas outra fermosura, outras divinas

graças de qu'esse sprito vejo cheio

as quais não dá, nem tira a natureza.

Il poeta del Lima è notevolmente vicino alla dolcezza fantastica del Petrarca, rinunciando alla vendicatività nelle terzine, presente invece in Camões, e optando per una lode imperitura delle grazie spirituali sovra-naturali di madonna. ${ }^{59}$

$E$ ancora il nostro tema, o per meglio dire il gesto, fu pure caro a Ronsard, ritrovandosi nelle Odes (A Ianne impitoiable, ed. I550: III, I3) e nei sonetti per Helene (II, 24):

O grand beaulté mais trop outrecuidée par les dons de Venus,

quand tu vérras ta face estre ridée et tes flocons cheneus,

contre le tens, et contre toi rebelle tu diras, en tansant:

«Que ne pensoi-ie alors que i'estoi belle, ce que ie va pensant,

ou bien pourquoi à mon desir, pareille ma pale ioüe n'est?

La beauté semble à la rose vermeille qui meurt si tost qu'ell'naist».

Voilà les vers tragiques, et la plainte, qu'au ciel tu envoiras, incontinent que ta face depainte par le tens tu voiras.

Tu sçais combien ardemment ie t'adore, indocile à pitié,

et tu me fuis, et tu ne veus encore te ioindre à ta moitié.

O de Paphos, et de Cypre régente déesse aus noirs sourcis, plus tost encor que le tens sois vangente mes dedignés soucis, et du brandon dont les cueurs tu enflammes des iumens tout au tour, brusle-la-moi, afin que de ses flammes ie me rie à mon tour. ${ }^{60}$ 
Quand vous serez bien vieille, au soir à la chandelle, assise auprès du feu, devidant et filant, direz, chantant mes vers, en vous ésmerveillant: «Ronsard me célébroit du temps que j'éstois belle».

Lors vous n'aurez servante oyant telle nouvelle, desja sous le labeur à demy sommeillant, qui au bruit de Ronsard ne s'aille resveillant, bénissant vostre nom de louange immortelle.

Je seray sous la terre, et fantaume sans os par les ombres Myrtheux je prendray mon repos; vous serez au fouyer une vieille accroupie, regrettant mon amour et vostre fier desdain. Vivez, si m'en croyez, n'attendez à demain: cueilllez dés aujourdhuy les roses de la vie. ${ }^{6 \text { I }}$

L'ode ronsardiana è riccamente classicheggiante, e quindi fisiologicamente aggressiva nei confronti della spietata donna; l'invocazione a Ciprigna è debitrice di Orazio, Carm. III, 26, 9-I2 ( $\ll 0$ quae beatam diva tenes Cyprum et / Memphin carentem Sithonia nive / regina, sublimi flagello / tange Chloen semel arrogantem»), ma non andrà mai dimenticato il carme del venosino contro Lydia senescente, terribile quant'altri mai (I, 25). Il sonetto a Elena, invece, celeberrimo, ba il torpore della veglia accanto alla fiamma e poi si crogiola nell'immaginazione del poeta stesso morto fra $i$ mirti, con un carpe diem finale ben poco petrarchesco. E impossibile non rammentare che il sonetto ronsardiano sarà imitato stupendamente da Yeats nella sua lirica giovanile When you are old (The Rose, I893). Un po' meno ovvio è il richiamo a una poesia di Pablo Neruda, anch'essa giovanile (da Crepusculario, I923), El nuevo soneto a Helena: "Cuando tu estés vieja, niña (Ronsard ya te lo dijo), / te acordarás de aquellos versos che yo decía» ecc. ${ }^{62}$ Qui l'amata, incanutita e avvizzita madre, sconterà con rimorso la ormai assoluta lontananza del poeta: «Yo estaré tan lejano» (v. 5, ribadito al $v .9$ e in chiusa al v. I4). Ma siamo in ambiti troppo distanti dal nostro assunto.

Questo regesto incompleto di occorrenze del topos sarebbe ancor più incompleto se non facessimo menzione dei sonetti di Shakespeare, in cui il motivo del tempo divoratore della bellezza è quasi ossessivo e strutturante, soprattutto nella sezione per il fair youth. Bisogna però dire che la declinazione scespiriana è intimamente diversa da quella degli 
esemplari fin qui scrutinati, formanti una trafila piuttosto coerente $e$ interconnessa. L'elegia dello sfiorire della gioventù smagliante e quindi dell'insorgere di melanconiche rughe e rovine sui campi floridi del volto non è pretesto a una ripicca vendicativa o a un mero vagheggiamento di tenerezze senili. L'obbligo è quello di fortificarsi (fortify, o anche ensconce) contro il tempo, ad esempio confidando nell'eternità della poesia: ${ }^{63}$

Against my love shall be as I am now, with Time's injurious hand crushed and o'erworn, when hours have drained his blood and filled his brow with lines and wrinkles, when his youthful morn hath travail'd on to age's steepy night, and all those beauties whereof now he's king are vanishing or vanished aout of sight, stealing away the treasure of his spring; for such a time do I now fortify against confounding Age's cruel knife, that he shall never cut from memory my sweet love's beauty, though my lover's life.

His beauty shall in these black lines be seen, and they shall live, and he in them still green.

Ancora, analogamente, nel son. 60 (vv. 9-I4):

Time doth transfix the flourish set on youth, and delves the parallels in beauty's brow, feeds on the rarities of nature's truth, and nothing stands but for his scythe to mow.

And yet to times in hope my verse shall stand, praising thy worth, despite his cruel hand.

E si potrebbero moltiplicare le citazioni, dal son. 55, ad es., o dal son. I8 Shall I compare thee to a summer's day?, in cui l'eterna estate del bel giovane splenderà perenne nei versi, o dal seguente I9, Devouring Time, in cui il poeta supplica il tempo di non incidere linee sulla fronte dell'amato ma poi conclude: «Yet do thy worst, old Time; despite thy wrong, I my love shall in my verse ever live young». Per non dire dell'altra strategia di salvaguardia dalla consunzione definitiva, quella 
del generare prole, nella serie cosiddetta "matrimoniale" dei sonn. I-I7. L'aggressività, insomma, che abbiamo visto marcare originariamente il locus dell' amata che invecchia, non si affaccia in Shakespeare se non in un occasionale monito, reperibile proprio nell' ultimo sonetto della grande sequenza per il fair youth: "Yet fear her [scil. the Nature], O thou minion of her pleasure, / she may detain, but not still keep her treasure» ecc. (I26, 9-IO).

Roberto Gigliucci 
I. Cfr. Petrarquismo plural e petrarquismo de koinè, in Petrarca 700 anos, a cura di Rita Marnoto, Coimbra, Instituto de Estudios Italianos da Faculdade de Letras da Universidade de Coimbra, 2005, pp. I 2 I-I 29, poi in versione italiana: Appunti sul petrarchismo plurale, «Italianistica», xxxiv, 2, 2005, pp. 71-75; Antipetrarchismo interno o petrarchismo plurale?, in Autorità, modelli e antimodelli nella cultura artistica e letteraria tra Riforma e Controriforma, a cura di Antonio Corsaro, Harald Hendrix, Paolo Procaccioli, Manziana (Roma), Vecchiarelli, 2007, pp. 9I-ı I. Vedi poi: Stefano Jossa, Simona Mammana, Petrarchismo e petrarchismi, in Nel libro di Laura, a cura di Luigi Collarile e Daniele Maira, Basel, Schwabe, 2004, pp. 91-107; Giorgio Forni, Pluralità del petrarchismo, Pisa, Pacini, 20 I I.

2. Il mio piccolo apporto in merito: $A$ realidade da literatura europeia, «Estudios italianos em Portugal», n.s., 2, 2007, pp. 399-41o, poi in versione italiana: Realtà della letteratura europea, in La letteratura italiana a Congresso. Bilanci e prospettive del decennale (1996-2006), a cura di Raffaele Cavalluzzi, Wanda De Nunzio, Grazia Distaso, Pasquale Guaragnella, Lecce, Pensa, 2008, tomo I, pp. 95-104.

3. Rimas a cura di Álvaro J. Da Costa Pimpão, Coimbra, Almedina, 2005, ultima ristampa.

4. Ivi, p. I 39 .

5. Garcilaso de la Vega, Poesie complete, vol. I, Liriche, a cura di Mario di Pinto, Napoli, Liguori, 2004 .

6. Cit. in Sonetos de Camões, a cura di Cleonice Serôa da Motta Berardinelli, Braga, Barbosa \& Xavier, I980, p. 567.

7. Compare nell'ediz. del ' 48 delle Rime; precedentemente in Rime diverse [...], Venezia, Giolito, i 545 (vd. Pietro Bembo, Le rime, a cura di Andrea Donnini, Roma, Salerno ed., 2008, pp. 358-36I).

8. Prima dell'ediz. postuma del 's 8 era comparso nel Libro quarto delle Rime di diversi [...], Bologna, Giaccarello, i 55 I, p. 82 (vd. Giovanni Della Casa, Rime, a cura di Stefano Carrai, Torino, Einaudi, 2003, pp. Iо9- I I 2). Naturalmente furono in molti a imitare il son. bembino; cito soltanto Benedetto Varchi, Quanto 'l pastor di Troia nel colle ideo.

9. Gutierre de Ceetina, Sonetos y madrigales, a cura di Begoña López Bueno, Madrid, Cátedra, i990, pp. 21 2-213.

ıo. In un altro sonetto dei Flores Diogo ripete il luogo comune della concentrazione di ogni bellezza naturale in un'unica donna: «Em fim em ti juntou a natureza / quanto reparte em mil, e em mil annos / com mil, e mil, e todas mui fermosas» (son. xxxviI, I 2-I4). Vd. l'ediz. moderna: Diogo Bernardes, Obras completas, a cura di Marques Braga, vol. I, Rimas varias Flores do Lima, Lisboa, Livraria Sá da Costa, i945.

i i. Panfilo Sasso, Sonetti (I-250), a cura di Massimo Malinverni, Pavia, Croci, 1996.

I 2. Sporadici ess. soprattutto quattrocenteschi, vd. Malinverni p. 93 ediz. cit., nota al son. 73 El capo hai biondo e avolto in or filato. 
i 3. Chiara Matraini, Rime e lettere, a cura di Giovanna Rabitti, Bologna, Commissione per i testi di lingua, 1989 .

i4. Berardino Rota, Rime, a cura di Luca Milite, Parma, Guanda, 2000.

i s. Bernardo Tasso, Rime, a cura di Domenico Chiodo e Vercingetorige Martignone, Torino, RES, 1995 .

i6. Benedetto Varchi, Opere, Trieste, Lloyd Austriaco, i 859.

17. Ediz. Costa Pimpão cit. p. 3 I 8.

18. Ivi, p. I 26.

19. Naturalmente idea-Idea-dea-Citerea secondo la grafia delle stampe e dei mss.

20. Costa Pimpão p. i 2 I. Cfr. Roger Bismut, La lyrique de Camões, Presses Universitaires de France, 1970, pp. 30-31.

2 I. Rita Marnoto, O petrarquismo portoguês do Renascimento e do Maneirismo, Univ. de Coimbra, I997, p. 525 ; Ead., Sete ensaios camonianos, ivi 2007, pp. 65 sgg.

22. Rimas varias de Luis de Camões [...] commentadas por Manuel de Faria y Sousa, Lisboa I685, rist. anast. ivi, INCM, I 972.

23. N. 9 ediz. Costa Pimpão p. I 2 I.

24. Vv. 37-38 ediz. Costa Pimpão p. 270.

25. Vv. 7-8; ediz. Costa Pimpão p. I 87. Vd. il bel commento all'ode in Liriche di Luís de Camões a cura di Silvio Pellegrini, Modena, Società Tipografica Modenese, I95 I, Pp. IO2-IO4.

26. Francesco Petrarca, Rime disperse, a cura di Angelo Solerti, Firenze, Sansoni, I919, pp. 37-40.

27. Antonio Tebaldeo, Rime, a cura di Tania Basile e Jean Jacques Marchand, Ferrara-Modena, ISR-Panini, I989-1992.

28. Matteo Maria Boiardo, Amorum libri tres, a cura di Tiziano Zanato, Torino, Einaudi, 1998.

29. Girolamo Parabosco, Il primo libro dei madrigali. isst. A cura di Nicola Longo, Roma, Bulzoni, I987.

30. «O mesmo Diogo Bernardes, na Écloga XIII (Piscatoria: Lilia e Meliso), escreve: "Lembrate a fermosura de Narciso, / que tal paga lhe deu seu desamor" (vv. 3233)»: Barbara Spaggiari, Camões e o Outono do Renascimento, Coimbra, Centro Interuniversitário de Estudos Camonianos, 20 I , p. ıо I n. 8, ma si veda tutto il bel saggio O mito de Narciso num soneto de Diogo Bernardes, ivi pp. 93-103. 
3 i. Luigi Alamanni, Versi e prose, a cura di Pietro Raffaelli, 2 voll., Firenze, Le Monnier, I 859 .

32. Ad esempio ficiniano, cfr. Marnoto, Sete ensaios camonianos, cit., pp. I 3 sgg.

33. Nella Giannetto, Il motivo dell" "amata incanutita" nelle rime di Petrarca e Boccaccio, in Miscellanea di studi in onore di Vittore Branca, II, Firenze, Olschki, I983, pp. 23-49. Vd. anche Ilaria Tufano, "Quel dolce canto». Letture tematiche delle rime di Boccaccio, Firenze, F. Cesati, 2006; Patrizia Bettella, The ugly woman, Toronto-BuffaloLondon, Univ. of Toronto Press, 2005, pp. I 5 2-1 53.

34. Leodegário A. de Azevedo Filho, Lirica de Camões, i, História, metodologia, corpus, Lisboa, Imprensa Nacional - Casa da Moeda, 1985.

35. Leodegário A. de Azevedo Filho, Lirica de Camões, 2. Sonetos, tomo II, Lisboa, Imprensa Nacional - Casa da Moeda, I989, pp. 82 I sgg. Noi riportiamo il sonetto nella redazione offerta dall'ediz. Costa Pimpão, cit., p. I 24, che segue la tradizione a stampa (I 595 e I 598 ).

36. Da Poeti del Cinquecento, Tomo I, Poeti lirici, burleschi, satirici e didascalici, a cura di Guglielmo Gorni, Massimo Danzi e Silvia Longhi, Milano-Napoli, Ricciardi, 200 I, p. I42, nell'edizione del testo i 530 a cura del compianto Gorni.

37. Per qualche riscontro con il rispetto di Poliziano Deh non insuperbir per tuo belleza vd. Giannetto, Il motivo dell"«amata incanutita», cit., pp. 4I sgg.

38. «O crudelis adhuc et Veneris muneribus potens, /insperata tuae cum ueniet pluma superbiae /et, quae nunc umeris inuolitant, deciderint comae, / nunc et qui color est puniceae flore prior rosae / mutatus Ligurinum in faciem uerterit hispidam, / dices, "Heu!" quotiens te speculo videris alterum: / "Quae mens est hodie, cur eadem non puero fuit, / vel cur his animis incolumes non redeunt genae? "».

39. Da Francesco Petrarca, Canzoniere, a cura di Marco Santagata, Milano, Mondadori, 1996, p. 55 .

40. Alcune cose sull'antipetrarchismo, «Critica letteraria» XxхviII, 2010 , 2, pp. 2 I I-225.

4i. Antonio Tebaldeo, Rime, II, i, Rime della vulgata, a cura di Tania Basile, Ferrara-Modena, ISR-Panini, I 992, p. 228. Per un commento e rimandi intertestuali vd. II, 2, Commento, pp. I 2 I-I 22. Si veda anche il son. Non seranno i capei sempre d'or fino (pp. I41-142), sul motivo del carpe diem.

42. Giovanni Boccaccio, Tutte le opere, vol V, tomo I, a cura di Vittore Branca, Milano, Mondadori, I992, pp. 52-53, 103.

43. Citiamo da Il sesto libro delle rime di diversi eccellenti autori [...], Venezia, al segno del Pozzo, I553, c. I 30 r. Il sonetto ricompare in I fiori delle rime de poeti illustri nuovamente raccolti e ordinati da Girolamo Ruscelli, Venezia, G.B. e M. Sessa, I 598 (poi I 569, I 579, I 586) e in Scelta nuova di rime de' più illustri et eccellenti poeti dell'età nostra, 
Venezia, G. Simbeni, i 573. Devo le indicazioni a Monica Bianco, curatrice dell'edizione critica delle rime di Venier ancora inedita.

44. Vd. Antonio Mezzabarba, Rime, a cura di Claudia Perelli Cippo, introduz. di Domenico Chiodo, Torino, Res, zoro, da cui citiamo (sonn. XxxIX-XL).

45. Vd. Chiodo, introduz. all'ediz. cit., pp. viii-ix e xvii-xviii; Monica Gori, Profilo di Antonio Isidoro Mezzabarba, «Studi italiani», XII, 2000, 2, pp. I 39-170: 142 sgg., I $52 \mathrm{sgg}$.

46. Un po' meno insolito ad es. nel caso dell'incresparsi delle acque, come nello stesso Mezzabarba: «crespano l'aure l'onde», son. Lxxxi ediz. cit. p. 57.

47. Citiamo da: Gaspara Stampa - Veronica Franco, Rime, a cura di Abdelkader Salza, Bari, Laterza, I9I 3, p. 200; il sonetto fu pubblicato nel i 550 nel Libro terzo delle rime di diversi nobilissimi et eccellentissimi autori (Venezia, al segno del Pozzo), c. $23 r$.

48. Milano, Longanesi, $1984^{2}$, p. 75 .

49. Son. anch'esso presente nel Libro terzo..., cit., c. 2iv. Mezzabarba invece declina il locus della brevità della vita in forme più epicuree, diciamo, e catulliane, anche se il "catullismo" di un sonetto come Lietta, il tempo vola: ridi e scherza è direttamente mediato dal Bembo latino (son. LXXviI, ediz. cit. p. 55; cfr. Gori, Profilo di Antonio Isidoro Mezzabarba, cit., pp. I61-162).

50. Peraltro presente in uno dei sonetti attribuiti a Petrarca, leggibile nell'aldina del i 5 I 4 e nella giuntina del I 522 , proprio in fin di verso come nel son. camoniano: «pur sarà mia vendetta il suo languire» (Rime disperse di Francesco Petrarca, a cura di Angelo Solerti, Firenze, Sansoni, 1909, son. cxxxviI Se sotto legge, Amor, vivesse quella, p. 201).

5 I. Si pensi a dittologie in fine verso come alte rapine o disdegna e sprezza, care al Tasso, ma anche la prima a un Celio Magno, la seconda (nella forma sdegna e sprezza) a un Galeazzo di Tarsia ecc.

52. Si cita da G. Guidiccioni - F. Coppetta Beccuti, Rime, a cura di Ezio Chiorboli, Bari, Laterza, I 9 I 2, p. 254.

53. Per il concetto complesso di traduzione-imitazione in Herrera vd. Inmaculada Osuna, Eva Redondo, Bernardo Toro, Las traducciones poéticas en las «Anotaciones» de Herrera, in Las «Anotaciones» de Fernando de Herrera. Doce estudios, a cura di Begoña López Bueno, Universidad de Sevilla, I 997, pp. 201-227: 2 I o sgg.

54. Da cui citiamo: Obras de Garcilasso dela Vega con anotaciones de Fernando de Herrera, en Sevilla, por Alonso de la Barrera, I 580 , pp. I 82 - 183 ; cfr. F. de Herrera, Obra poética, ediz. critica a cura di José Manuel Blecua, vol. I, Madrid, Anejos del Boletín de la Real Academia Española, 1975, p. 270.

55. Vd. Garcilaso de la Vega, Poesie complete, vol. I, Liriche, cit., son. XXIII, p. i i 8. 
56. Il son. è anche in De le rime di diversi nobili poeti toscani, raccolte da M. Dionigi Atanagi, libro secondo, Venezia, L. Avanzo, 1565, c. 136r. Vd. poi Rime di Jacopo e Tommaso Mocenighi fratelli e gentiluomini veneziani. Ora per la prima volta raccolte da Giovanni Alvise Mocenigo patrizio veneziano, Brescia, Giammaria Rizzardi, 1756.

57. Rimas varias de Luis de Camões, cit., t. I, pp. I I9-I 23.

58. Ivi, p. I 22.

59. Per questa analisi rimando a Rita Marnoto, O petrarquismo portoguês do Renascimento e do Maneirismo, cit., pp. 53 I-532.

6o. Da Les quatre premiers livres des odes de P. de Ronsard [...], Paris, Chez Guillaume Cavellart, MDL, cc. 87v-88r. Thomas Stanley tradusse-imitò l'ode ronsardiana nel secolo seguente, come ha dimostrato Mario Praz, Ricerche anglo-italiane, Roma, Edizioni di Storia e Letteratura, I 944, pp. I I I sgg.

6i. Pierre de Ronsard, Les Amours, a cura di Marc Bensimon e James L. Martin, Paris, Garnier-Flammarion, I98 I, p. 298.

62. Vd. Pablo Neruda, Crepuscolario, a cura di Giuseppe Bellini, Firenze, Passigli, 2004, p. 28.

63. Citiamo da William Shakespeare, Sonetti, a cura di Alessandro Serpieri, Milano, Rizzoli, I995'; si tiene anche presente Shakespeare's Sonnets, a cura di Stephen Booth, New Haven and London, Yale Univ. Press, I 977. 